\title{
Neuroprotective Agent
}

National Cancer Institute

\section{Source}

National Cancer Institute. Neuroprotective Agent. NCI Thesaurus. Code C1509.

A pharmacological agent that protects the central nervous system from damage caused by acute ischemia or chronic neurodegenerative diseases. 